Service social

\title{
Une recherche exploratoire sur deux expériences de familles d'accueil de réadaptation
}

\section{Mario Paquette}

Volume 36, numéro 1, 1987

L'informatique dans les services sociaux

URI : https://id.erudit.org/iderudit/706346ar

DOI : https://doi.org/10.7202/706346ar

Aller au sommaire du numéro

Éditeur(s)

École de service social de l'Université Laval

ISSN

1708-1734 (numérique)

Découvrir la revue

Citer ce document

Paquette, M. (1987). Une recherche exploratoire sur deux expériences de familles d'accueil de réadaptation. Service social, 36(1), 148-159.

https://doi.org/10.7202/706346ar
Résumé de l'article

Une recherche sur les familles d'accueil s'impose au moment où les structures intermédiaires deviennent très populaires auprès de ceux qui favorisent la désinstitutionnalisation. Dans une démarche à caractère exploratoire, on tente de faire le point sur deux expériences de familles d'accueil de réadaptation. Des données, qui ont fait l'objet d'une analyse de contenu qualitative, ont été recueillies auprès de bénéficiaires, de responsables de familles d'accueil, de parents de familles naturelles et de quelques intervenants sociaux. Plusieurs suggestions, visant prioritairement la famille d'accueil, sont énoncées. 
Paquetre, Mario, agent de planification et de recherche au Département de santé communautaire du Centre hospitalier régional de Lanaudière, à Joliette.

\section{Une recherche exploratoire sur deux expériences de familles d'accueil de réadaptation 1}

\section{Mario Paquette}

Au moment où l'on présente de plus en plus les structures intermédiaires comme des alternatives à l'institution, il est important de se pencher sur le fonctionnement de telles structures là où elles existent. Ici, nous examinons le cas des familles d'accueil de réadaptation en confrontant les points de vue, tant des bénéficiaires, des travailleurs sociaux impliqués dans leur placement que des familles d'accueil et familles naturelles. Ainsi, cette démarche permet de dégager une vision d'ensemble de la réalité entourant le vécu de ce type d'alternative.

Le présent article rend compte d'une recherche exploratoire effectuée dans la région de la Côte-Nord, où l'auteur occupait un poste d'agent de recherche au Conseil régional de la santé et des services sociaux. Il comporte deux parties : l'exposé du contexte, de l'objectif et de la méthodologie, et la présentation des résultats. ${ }^{2}$

\section{Contexte, objectif et méthodologie}

La ressource famille d'accueil de réadaptation ${ }^{3}$ a été mise sur pied dans cette région suite à la décision de fermer un foyer de groupe où habitaient huit déficients mentaux adultes. Les responsables de la première famille d'accueil de réadaptation étaient des éducateurs spécialisés. Un d'entre eux occupait antérieurement un poste au foyer de groupe. Cette famille a commencé à recevoir une partie de sa clientèle en septembre 1982, mais a mis fin à l'expérience un an plus tard. Suite à ce désistement, une autre famille d'accueil de réadaptation a été recrutée. 
Ainsi, le mandat de notre recherche consistait à faire le point sur ces deux expériences de familles d'accueil de réadaptation, et était défini ainsi :

«À partir des différentes personnes impliquées par la création des deux familles d'accueil de réadaptation dégager leur opinion, leur perception bref ce qu'ils pensent de ces deux expériences vécues jusqu'à maintenant ". ${ }^{4}$

Comme l'objectif était de circonscrire, dans les faits, une certaine réalité et non de vérifier une ou des hypothèses, notre démarche en fut une à caractère exploratoire qui s'inscrivait dans une approche inductive où des données qualitatives ont constitué le corpus des informations recueillies.

L'entrevue individuelle semi-structurée a été choisie comme méthode de cueillette des données. Au total onze entrevues ont été réalisées auprès des personnes suivantes : trois bénéficiaires, les responsables des deux familles d'accueil, les parents de deux familles naturelles et quatre intervenants. Un schéma d'entrevue a été élaboré pour chaque sous-catégorie de la population à l'étude.

La cueillette des données s'est effectuée entre les mois de janvier et mars 1985, au domicile des familles naturelles et de la deuxième famille d'accueil. La première famille d'accueil, les intervenants et les bénéficiaires ont été rencontrés dans leurs milieux de travail respectifs. Les autorités responsables des bénéficiaires nous ont confirmé que ceux-ci étaient aptes à répondre aux questions formulées. Toutefois, c'est une éducatrice proche de ces derniers qui a réalisé toutes les entrevues, auxquelles nous avons assisté à titre d'observateur. Toutes ont été enregistrées avec un magnétophone et, par la suite, retranscrites intégralement. Les données recueillies ont fait l'objet d'une analyse de contenu qualitative, selon la méthode présentée par Chiglione et Matalon. ${ }^{5}$ Nous présentons ici les résultats par groupe d'acteurs.

\section{Résultats de la recherche ${ }^{6}$}

\section{Les bénéficiaires}

\section{Perception face à leur milieu de vie}

Deux bénéficiaires affirment qu'ils aiment vivre en famille d'accueil de réadaptation. Les propos du troisième sont plus nuancés: si son discours laisse présager une certaine insatisfaction, celle-ci semble être 
liée davantage à l'endroit qu'il habite plutôt qu'au fait de vivre en famille d'accueil. Tous ont cependant dit s'y sentir "chez eux".

\section{Perception de leurs activités (travail, loisirs)}

Étant donné qu'ils doivent se rendre au travail 7 durant la semaine, en suivant un horaire bien précis, il va sans dire que cette activité occupe la majeure partie du temps passé à l'extérieur de la famille d'accueil. D'ailleurs on retrace, dans le discours des répondants, un intérêt marqué pour le travail.

En ce qui regarde les loisirs, tous aiment les passe-temps, à l'intérieur ou à l'extérieur de la famille d'accueil. À l'intérieur, le principal loisir consiste à regarder la télévision et écouter la radio. Même si on ne peut pas nécessairement les considérer comme passe-temps, l'exécution de certaines tâches ménagères (faire sa chambre, la vaisselle, etc.) fait partie intégrante du quotidien. Pour ce qui est des loisirs extérieurs, ils se composent principalement d'activités de plein air (sortir dehors, marcher, faire du ski de fond).

Un problème important semble préoccuper les bénéficiaires: ils souffrent de leur isolement social. On a remarqué que les activités à caractère socio-culturel et communautaire étaient peu courantes. Ce problème n'est pas lié à la famille d'accueil mais à l'isolement géographique du lieu de résidence de cette dernière. Comme il n'y a pas de transport en commun et qu'il existe peu de services communautaires (d'ailleurs éloignés), il est pratiquement impossible de faire des activités à l'extérieur sans devoir compter sur la famille d'accueil qui, elle, possède un véhicule.

\section{Les familles d'accueil de réadaptation}

\section{Perception des aspects positifs et négatifs de l'expérience}

L'arrivée d'un quatrième ${ }^{8}$ bénéficiaire plus jeune, n'ayant pas le même degré d'autonomie que les autres, a exigé beaucoup d'énergie selon une famille d'accueil. Ce surinvestissement a affecté non seulement l'intervention auprès des autres, mais aussi les comportements de ces derniers puisqu'on s'occupait moins d'eux. À la lumière de cette expérience, les familles d'accueil en arrivent à insister sur la nécessité de I'homogénéité des bénéficiaires en termes d'autonomie, d'âge et de comportement. 
La deuxième famille d'accueil a souligné qu'elle se sentait privée de moyens pour arriver, quand cela s'impose, à modifier les comportements des bénéficiaires, d'où une attitude de résignation face à ceux-ci et de défaitisme face à l'intervention. La première famille d'accueil se sentait moins démunie face à leurs comportements. Cela s'explique peut-être par le fait que les responsables avaient une formation en éducation spécialisée. Quoi qu'il en soit, la deuxième famille n'en observe pas moins les bénéficiaires avec soin ; de plus, elle analyse énormément les comportements de ceux-ci : "C'est un surplus d'énergie qu'il a [...] Lui il est plus sensible d'après ce que je peux voir. " Ainsi, une connaissance intime de sa clientèle favorise des interventions dont la famille ne reconnaît pas toujours l'efficacité au niveau de la réadaptation :

"J'ai appris à ne pas courir après [...] parce que durant le temps qu'il s'éloigne pis qu'il va prendre une marche, on dirait qu'il a le temps de penser, de reviser ça, pis il revient [...]"

Signalons, dans un autre ordre d'idée, que le rôle de famille d'accueil n'est pas sans influencer la vie du couple sur le plan de l'intimité étant donné la place qu'occupent les bénéficiaires dans la vie de tous les jours :

"Nous autres on vit un petit peu des moments difficiles parce que notre mariage s'en ressent de cet état-là [...] Ils sont tellement accaparents [les bénéficiaires], on n'a plus d'intimité [...] Nos moments il faut les calculer [...] II faut être là pour eux autres pis après ça pour nous autres."

Cependant, malgré certains problèmes rencontrés par les deux familles d'accueil, la première avoue que l'expérience lui a démontré qu' " il faut savoir foncer " pour être en mesure de résoudre les problèmes qu'impliquent les responsabilités d'une famille d'accueil de réadaptation. De plus, la présence des bénéficiaires vingt-quatre heures par jour permet de voir leur progrès dans l'acquisition d'une plus grande autonomie, et ce, davantage que pendant un horaire normal de travail. Enfin, cette famille d'accueil a exprimé qu'il s'établit une relation entre elle et les bénéficiaires, laquelle permet de se sentir "près " les uns des autres; relation qu'il n'est pas possible de vivre en n'importe quel milieu de travail.

\section{Degré de satisfaction face aux services dispensés par les établissements}

Bien que les deux familles fussent au courant des services offerts par les établissements (soutien d'éducateur, travailleur social), les entre- 
tiens ont révélé, cependant, un manque de communication et de support des établissements.

\section{Qualités personnelles requises}

La principale qualité dont la famille d'accueil de réadaptation devrait faire preuve, c'est d'être capable de favoriser la communication avec les différents milieux de vie des bénéficiaires (milieu de travail, famille naturelle). Cette communication est essentielle afin de permettre une plus grande compréhension des problèmes rencontrés de part et d'autre avec les bénéficiaires (par exemple au niveau des comportements). Pour la première famille d'accueil, les personnes ne doivent pas seulement être considérées comme des pensionnaires en "chambre et pension". On doit leur apporter une écoute attentive et «prendre le temps de vivre" avec elles. La deuxième complète cette affirmation en disant que : "Ça prend beaucoup de patience pis de soins [...] [et] une bonne psychologie [...] En réalité, il faut avoir été parent ».

\section{Encadrement des bénéficiaires}

Les familles d'accueil sont catégoriques : les bénéficiaires se sentent vraiment heureux dans le cadre de vie qu'elles leur offrent. Une journée en famille d'accueil se déroule ainsi: pour les jours de la semaine, on se lève tôt afin de se préparer pour aller travailler. Le soir, chacun est libre de faire ce que bon lui semble. Outre les activités faites à l'extérieur de la maison, les fins de semaine sont consacrées au repos.

Dans la première comme dans la deuxième famille, une bonne partie des temps libres est consacrée à l'exécution de tâches ménagères. Ainsi, l'encadrement offert correspond, aux dires de celles-ci, à celui d'une famille ordinaire.

Par ailleurs, si les responsabilités de la première famille d'accueil envers les bénéficiaires pouvaient se définir beaucoup plus en termes d'atteinte d'objectifs de réadaptation, la deuxième envisage ces responsabilités en termes d'obligation. On se sent hyperresponsable et conscient des erreurs potentielles:

"[...] Si tu fais une erreur quelque part [...] pis la moindre petite affaire qui pourrait arriver, t'aurais tort [...] On est conscient que des choses de même ça pourrait nous arriver. Ça nous fait peur un peu ça [...]"

D'ailleurs, elle avoue trouver difficile d'assumer pleinement son rôle. 
D'autre part, même si les deux familles n'ont pas perçu un besoin de formation, la première a tout de même précisé qu'il était nécessaire d'avoir une formation minimale pour être en mesure d'atteindre les objectifs fixés par les instances responsables des bénéficiaires. Quant à la deuxième, pour se valoriser, elle aurait apprécié suivre une formation si elle avait été dispensée. De plus, elle considérait important d'avoir de l'information de base sur la clientèle (traits de caractère, comportements, personnalité, etc.).

Les deux familles ont manifesté le désir de rencontrer périodiquement d'autres familles d'accueil. Une de celles-là considérait que cette activité pouvait favoriser des échanges fructueux en regard des moyens utilisés pour résoudre les problèmes et aussi constituer une occasion de se ressourcer.

\section{Relation avec les parents des bénéficiaires}

Il n'est jamais survenu de conflits majeurs avec les parents. Cependant, le type de relations entretenues avec les parents n'a été qualifié que de "relation téléphonique", symptôme d'un manque de communication entre les parties.

\section{Périodes d'inactivité (congés)}

Si on se rappelle les difficultés rencontrées par la deuxième famille, on comprend mieux leur désir de se ressourcer régulièrement. Toutefois, si les périodes de répit sont moins vitales à la première famille, de par sa composition, qui permet aux responsables de se libérer régulièrement, il en va tout autrement pour la deuxième. Effectivement, pour elle, l'existence de périodes de congés est l'exception plutôt que la règle.

\section{Les familles naturelles}

Relation entre la famille d'accueil et les parents des bénéficiaires

Bien que les relations entre la famille d'accueil et les parents des bénéficiaires soient cordiales, on dénote tout de même un problème à un autre niveau. En effet, les parents n'acceptent pas le fait que leurs enfants vivent en périphérie à la suite de directives venant de l'organisme responsable du placement. 
Collaboration des parents dans le projet de la famille d'accueil

Les deux familles naturelles considèrent important de collaborer avec la famille d'accueil. À leur avis, cette collaboration doit prendre la forme d'un soutien, par exemple: aller chercher régulièrement leur enfant, tout en maintenant une communication entre les parties pour qu'elles puissent prendre ensemble les décisions nécessaires quand cela l'exige. C'est de cette manière que les familles naturelles disent collaborer actuellement.

Perception des parents face au milieu de vie de leur enfant

Les familles naturelles sont satisfaites parce que l'on considère que leur enfant est dans une "bonne" famille d'accueil. On est d'ailleurs convaincu qu'il est heureux à l'intérieur de celle-ci.

Par ailleurs, il est important d'indiquer qu'une famille naturelle a la nette impression que son enfant avait une meilleure formation au foyer de groupe et avec la première famille d'accueil. En effet, la deuxième n'aurait pas eu la formation nécessaire pour travailler auprès de cette clientèle. Même si l'on croit que celle-ci remplit bien les objectifs d'une famille d'accueil ordinaire, on doute sérieusement de sa capacité de fournir des moyens de réadaptation.

Pour'sa part, l'autre famille naturelle a aussi mis en doute cette capacité de réadaptation. Mais, dans ce cas, ce n'est pas uniquement la famille d'accueil qui est visée mais l'environnement social dans lequel vit actuellement son enfant. Du reste, elles ont remarqué la perte d'autonomie de leur enfant depuis qu'il vivait avec la deuxième famille d'accueil.

Pour les parents, la ressource famille d'accueil est synonyme d'insécurité et d'instabilité. D'abord, l'expérience de la première n'a duré qu'une année. Ensuite, la deuxième a déménagé de la ville pour aller demeurer en périphérie. Enfin, on est conscient que ce n'est pas facile d'être famille d'accueil de réadaptation et que le recrutement d'une "bonne» famille d'accueil est très difficile étant donné la rareté des postulants. ${ }^{9}$

En outre, on anticipe un nouveau désistement. Pas surprenant, dans ces conditions, que cette ressource ait été qualifiée de "[...] solution à la bonne franquette [...] [de] maison de dépannage [...] [de] situation temporaire improvisée " où les enfants, en changeant continuellement de famille d'accueil, ne sont en fait que les " cobayes » d'une 
ressource n'offrant aucune garantie de stabilité. À vrai dire, si l'on résume les commentaires d'une famille naturelle, celle-ci a la conviction que son enfant, en vivant dans cette ressource, a "descendu d'une marche " par rapport au foyer de groupe. Selon elle, au foyer de groupe, les bénéficiaires étaient chez eux, dans "leur maison", dans "leurs affaires ", et à proximité de tout. Maintenant, ils ne se sentent pas chez eux, ils vivent dans la famille d'accueil et loin de tous les services.

\section{Commentaires relatifs à la fermeture du foyer de groupe}

Ce ne sont pas tant les raisons de la fermeture du foyer de groupe qui ont retenu ici notre attention, mais le fait que les familles naturelles n'ont pas été impliquées dans des décisions prises concernant leur enfant (comme celle de fermer le foyer de groupe). En effet, ce qui frappe dans les propos recueillis, c'est qu'elles ont l'impression d'être complètement en dehors des décisions. Lorsqu'elles sont consultées, c'est pour leur faire part de décisions déjà prises.

\section{Les intervenants}

\section{Définition de la famille d'accueil de réadaptation}

Premièrement, selon les intervenants, la famille d'accueil de réadaptation, comme toutes les familles d'accueil, doit répondre aux besoins essentiels des bénéficiaires (résidence, soins de base, nourriture, etc.). Ce qui la différencie d'une famille d'accueil ordinaire, c'est qu'elle devrait recevoir une clientèle plus « lourde ", moins autonome et nécessitant, par le fait même, un encadrement plus soutenu. Dans ses fonctions de réadaptation, ce type de famille doit viser, à partir d'un plan précis d'intervention élaboré conjointement par les établissements concernés, un objectif de développement du potentiel des bénéficiaires afin que ces derniers puissent s'intégrer à leur milieu. Elle n'a donc pas uniquement une fonction d'hébergement ou de "protection sociale", mais aussi, fondamentalement, de réadaptation.

\section{Perception des intervenants face à cette ressource}

Tous les intervenants se sont dit satisfaits des deux expériences vécues jusqu'à présent. Toutefois, selon eux, si la fonction de réadaptation 
est fondamentale dans les objectifs que devrait poursuivre la famille d'accueil de réadaptation, tous ne sont pas du même avis sur la réalisation de cette fonction. En effet, il y a trois perceptions à ce sujet : 1. la famille d'accueil répond aux besoins de réadaptation des bénéficiaires; 2 . elle ne fait pas de réadaptation, mais, en contrepartie, les bénéficiaires vivant au sein de la communauté profitent automatiquement d'une certaine forme de réadaptation; 3 . il est impossible pour une famille d'accueil de faire de la réadaptation.

En ce qui regarde l'avenir de cette ressource, on ne cache pas une certaine inquiétude : la famille d'accueil de réadaptation pourrait devenir une espèce de "fourre-tout " permettant une désinstitutionnalisation à outrance effectuée au nom d'une plus grande normalisation. ${ }^{10} \mathrm{En}$ fait, les intervenants entrevoient des possibilités intéressantes, mais également des limites qu'il s'agirait de circonscrire, car cette ressource ne constitue certainement pas la seule alternative possible.

De plus, ils sont unanimes quant à l'ampleur du problème de recrutement: la demande est plus forte que l'offre. Le problème est tellement manifeste qu'on nous a fait état de la chance qui s'est présentée lorsque d'anciens employés du foyer de groupe ont postulé pour devenir famille d'accueil de réadaptation.

Ce problème de recrutement constitue une limite importante du mouvement de désinstitutionnalisation dans lequel on a peut-être postulé trop rapidement que la société était d'emblée prête à s'inscrire.

Enfin, on a fait part du problème d'instabilité de cette ressource, qualifiée de "structure fragile": "L'expérience nous dit que ce sera toujours une structure fragile dans le sens qu'elle peut craquer à n'importe quel temps". Or, s'il a déjà été signalé que les familles naturelles souffraient d'un sentiment d'insécurité dû à cette instabilité, il en est de même pour les intervenants :

" Je reste inquiet parce que je peux avoir un téléphone demain ou dans une semaine pour dire ça ne marche plus."

Bref, en plus de la pénurie de ressources et du fait que tout le monde ${ }^{11}$ ne peut s'improviser famille d'accueil de réadaptation, on doit s'attendre, somme toute, à des désistements fréquents de la part des responsables.

\section{Soutien à la famille d'accueil}

Certains intervenants croient que les familles d'accueil de réadaptation n'ont peut-être pas eu tout le soutien (éducateur, travailleur social) qui leur était nécessaire. Cependant, il est possible d'expliquer 
les problèmes rencontrés. Par exemple, pour ce qui est du support d'un travailleur social, il est difficile de donner ce service sur une base régulière puisque le personnel affecté à ce type de ressources est en constante mobilité. En ce qui a trait au soutien d'un éducateur, les justifications amenées concernent surtout la première famille d'accueil. On peut résumer l'explication de cette source d'aide par la question suivante : comment peut-on être objectivement réceptif à une demande de soutien d'éducateur alors que les demandeurs sont eux-mêmes des éducateurs? D'autre part, si on regarde le soutien en dehors des interventions d'un éducateur ou d'un travailleur social, certains affirment que ce soutien est comparable à celui donné ailleurs dans la province.

Du reste, la participation des parents à une ressource comme la famille d'accueil de réadaptation est considérée des plus importantes et devrait, par le fait même, être favorisée.

\section{Conclusion}

Si l'on veut que la vocation de réadaptation, à l'intérieur des familles d'accueil, ne soit pas qu'un vœu pieux, il est impératif que certains constats dégagés ici ne soient pas laissés pour compte, comme I'isolement social des bénéficiaires, le manque de support accordé aux familles d'accueil; on qualifie ces ressources, dont il y a pénurie, de structures fragiles et insécurisantes. L'intégration sociale des personnes atteintes d'une déficience intellectuelle est tributaire des réponses que l'on apportera aux questions posées par ces constats. À cet égard, plusieurs auteurs ${ }^{12}, 13,14,{ }^{15}, 16$ ont déjà proposé des solutions dans le but d'assurer à ces personnes l'intégration sociale et des conditions de vie plus acceptables au sein de la communauté. Les résultats de notre recherche nous permettent d'apprécier la pertinence de plusieurs de ces solutions qui ont été énoncées et qui visent essentiellement la famille d'accueil. Par exemple, on suggère :

- un support et un encadrement accrus ;

- une formation minimale et du perfectionnement ;

- l'encouragement des échanges entre les familles d'accueil ;

- la création de mesures de répit ;

- la nécessité de favoriser l'implication des parents. 


\section{Notes et références}

1 Je remercie l'équipe de recherche du C.R.S.S.S. Côte-Nord, entre autres Danièle Francour, pour leurs critiques de la version finale de ce texte. Je tiens aussi à remercier particulièrement Denise Couture, professeure au Département de sociologie de l'Université de Montréal; les critiques formulées dans les différentes versions de ce texte sont garantes de l'énorme somme de travail qu'elle a investie.

2 Paquette, Mario, Évaluation de deux expériences de familles d'accueil de réadaptation, rapport de recherche, Conseil régional de la santé et des services sociaux de la Côte-Nord, 1987.

3 Étant donné que cet article traite spécifiquement de la famille d'accueil de réadaptation et que cette ressource est, du point de vue légal, relativement récente, il convient de présenter la définition retenue par le Ministère de la santé et des services sociaux,

"La famille d'accueil de réadaptation qui fournit à un maximum de quatre personnes un programme de réadaptation, selon un plan d'intervention établi pour une durée limitée pour chaque bénéficiaire par un établissement offrant des services de réadaptation et relié au Centre de Services sociaux par un contrat de services."

(Source : Ministère des affaires sociales, La catégorisation des familles d'accueil, principes directeurs et modalités organisationnelles et financières, Québec, M.A.S., Direction générale des programmes et des services sociaux, 1984, p. 4.)

4 Paquette, M., op. cit.

5 Chiglione, Rodolphe et Benjamin Matalon, les enquêtes sociologiques, théorie et pratique, Paris, Armand Colin, 1982, 301p.

6 Nous présentons dans cet article la plupart des thèmes étudiés dans notre recherche.

7 Suite à la réorientation des fonds que permettait la fermeture du foyer de groupe, une nouvelle structure ("atelier $138 »)$ a été mise sur pied pour les déficients mentaux de la région. Les bénéficiaires en question suivent à cet endroit un programme adapté d'intégration au travail.

8 La famille d'accueil de réadaptation peut accueillir un maximum de quatre personnes (voir note 3 ).

9 Ce point sera abordé plus longuement dans la partie consacrée aux intervenants.

10 Selon Wolfensberger dans: Laurendeau et al., "Une évaluation de l'impact d'un programme de désinstitutionnalisation de personnes vivant un handicap mental ", Déficience mentale, vol. 33, n०1,1983; 3-18; 45-46), la normalisation est: "L'utilisation de moyens culturellement normatifs (ou valorisés) afin d'offrir aux personnes handicapées des conditions de vie qui sont au moins aussi bonnes que celles du citoyen moyen et d'instaurer des comportements personnels, une apparence, un statut et une réputation qui se rapprochent le plus de ceux du citoyen moyen ".

$\grave{A}$ noter que, dans une nouvelle conceptualisation de la normalisation, il préconise désormais l'utilisation du terme "valorisation sociale" plutôt que "normalisation " et " principe de normalisation ». Pour en savoir davantage sur les fondements théoriques ayant conduit l'auteur à faire ce choix, consulter : 
Wolf WOLfensBerger, "La valorisation du rôle social : une nouvelle "conceptualisation de la normalisation ", Déficience mentale, vol. 34, n² 2, 1984: 26-30.

1 Selon les intervenants, les responsables de la famille d'accueil de réadaptation devraient avoir les qualités suivantes : être sûrs d'eux-mêmes, calmes, patients, tolérants, disponibles, dévoués, fermes, non impulsifs, avoir un niveau d'acceptation élevé, une forte tolérance à la frustration, un esprit familial et d'entraide, être capables de jugement, de dialoguer, de prendre du recul devant différentes situations, de favoriser un climat affectif, de ne pas voir les bénéficiaires comme des " cas " et le fait d'être famille d'accueil de réadaptation comme une " job", être cohérents dans les interventions, réceptifs au soutien extérieur et capables d'en demander quand cela est nécessaire.

12 BEAUDRY, Raymond, "Une expérience de famille d'accueil de réadaptation ", L'Accueil, vol. 12, no 2, 1985 : 44-48.

13 BLANCHET, André, "Les défis de la prochaine décennie ", Revue canadienne de déficience mentale, vol. 35, no 1, 1985 : 18-22, 34.

14 DorvIL, Henri, "Les ressources alternatives au C.H. Louis-H. Lafontaine", Administration hospitalière et sociale, vol. 30, $\mathrm{n}^{\circ} 6,1984: 31-41$.

15 MCLEAN, Jan, "Les services communautaires : peuvent-ils répondre aux besoins individuels", La revue canadienne de la déficience mentale, vol. $34, n^{\circ} 3$, 1984 : 10-18.

16 RaINVILLE, Manon et Denise LALANDE, "Les ressources non institutionnelles à l'enfance : le passé, le présent, l'avenir ",Administration hospitalière et sociale, vol. 30 , no $6,1984: 54-56$. 